\title{
Heterosis study in Okra [Abelmoschus esculentus (L.) Moench] genotypes for pod yield attributes
}

\author{
Sujit Kumar ${ }^{1 *}$, A. K. Singh ${ }^{2}$, Hirdesh Yadav ${ }^{1}$ and Alka Verma ${ }^{1}$ \\ ${ }^{1}$ Department of Vegetable Science, College of Agriculture, G.B.Pant University of Agriculture and Technology, \\ Pantnagar- 263145 (Uttarakhand), INDIA \\ ${ }^{2}$ Department of Horticulture, Banaras Hindu University, Varanasi-221005 (U.P.), INDIA \\ *Corresponding author. E-mail: sujittca5001@gmail.com
}

Received: August 2, 2016; Revised received: January 22, 2017; Accepted: April 17, 2017

\begin{abstract}
A study was conducted at Vegetable Research Farm, Department of Horticulture, Institute of Agriculture Sciences, Banaras Hindu University, Varanasi during Spring-Summer and Rainy season of 2012 and 2013 using 12 diverse parental lines of okra and their $66 \mathrm{~F}_{1}$ hybrids (through diallel cross-excluding reciprocals) with the objective to measure the extent of heterosis over better parent and standard commercial check varieties for the purpose of judging the extent up to which heterosis can be exploited in commercial okra breeding. The extent of heterosis for five best crosses over better parent and check (48.32\% to $82.42 \%$ and $7.13 \%$ to $35.66 \%$, respectively) for yield per hectare suggested the great scope of realizing higher yield in okra through heterosis breeding. Other economic traits also recorded moderate to high level of heterosis over the better parents. The cross combination IC $282280 \times E C$ - 329380showed high heterosis over better parent and standard check for pod yield $(82.42 \%$ and $35.66 \%)$, number of pods per plant $(62.82 \%$ and $48.54 \%)$ and respectively. This particular cross combination eventually resulted the height magnitude of heterobeltiosis and standard heterosis for the most of the desirable growth parameters as well as yield attributing characters which may be taken for further breeding programme.
\end{abstract}

Keywords: Diallel cross, Economic traits, Heterobeltiosis, Heterosis, Okra

\section{INTRODUCTION}

Okra [Abelmoschus esculentus (L.) Moench] known as bhindi or lady's finger is an important annual vegetable crop grown in the tropical and sub-tropical regions of world (Arapitsas, 2008; and Saifullah and Rabbani, 2009). Fairly good amount of proteins, carbohydrates, vitamins (A, B and C) and minerals is found in pod of Okra (Owolarafe, 2004; Gopalan et al., 2007; Arapitsas, 2008 and Dilruba et al., 2009) thus it plays an important role in human diet (Kahlon et al., 2007 and Saifullah and Rabbani, 2009). So many varieties have been developed in okra but significant increase in productivity potential could not be realized possibly because of ceiling in genetic potential of the genotypes. Therefore, there is an urgent need of genetic improvement of crop for yield which can be achieved by exploitation of hybrid vigour (heterosis). In most of developed country like Japan and USA, mainly $F_{1}$ hybrid varieties are cultivated on commercial scale instead of open pollinated varieties. Heterosis breeding programme aiming to develop a hybrid variety comprises identification of desirable parents which can produce $\mathrm{F}_{1}$ hybrid having high level of economic heterosis. The presence of great extent of natural variation for various characters among the varieties of okra suggested a good scope of improvement in the economic traits through heterosis breeding techniques. Keeping in view the above facts, the present study was planned and carried out using 12 parents and their $66 \mathrm{~F}_{1}$ hybrids (excluding reciprocals) with the objective to measure the extent of heterosis over better parents and standard commercial check varieties for purpose of judging the extent up to which heterosis can be exploited in commercial okra breeding.

\section{MATERIALS AND METHODS}

The present experiment conducted at Vegetable Research Farm, Department of Horticulture, Institute of Agriculture Sciences, BHU, Varanasi during Spring -Summer and Rainy season of 2012 and 2013. This place is located in south east part of Varanasi city at $25^{\circ} 15^{\prime}$ North latitude and $83^{\circ} 03^{\prime}$ East longitudes at an elevation of $129.23 \mathrm{~m}$ above the mean sea level. The average annual rainfall is about 1110 millimeter $(\mathrm{mm})$. The major portion of precipitation (about 85 to $90 \%$ ) is received during July to September. The soil of the experimental site contains $49.75 \%$ sand, $28.73 \%$ silt and $21.52 \%$ clay. The available soil nitrogen, phosphorous and potassium status have been recorded as $0.082,0.126$ and $0.640 \%$, respectively. The experimental material consisted 12 diverse varieties/lines (thereafter called genotypes) of okra, provided by the Indian Institute of Vegetable Research, Varanasi 
Table 1. Genotypes of okra used in the present experiment.

\begin{tabular}{lcc}
\hline Parents & Name of the genotypes & Sources \\
\hline $\mathrm{P}_{1}$ & Larm -1 & IIVR-Varanasi \\
$\mathrm{P}_{2}$ & IC -282280 & IIVR-Varanasi \\
$\mathrm{P}_{3}$ & IC -282337 & IIVR-Varanasi \\
$\mathrm{P}_{4}$ & IC -128891 & IIVR-Varanasi \\
$\mathrm{P}_{5}$ & IC -111527 & IIVR-Varanasi \\
$\mathrm{P}_{6}$ & EC -329380 & IIVR-Varanasi \\
$\mathrm{P}_{7}$ & IC -282279 & IIVR-Varanasi \\
$\mathrm{P}_{8}$ & VRO -5 & IIVR-Varanasi \\
$\mathrm{P}_{9}$ & IC -329422 & IIVR-Varanasi \\
$\mathrm{P}_{10}$ & IC -18537 & IIVR-Varanasi \\
$\mathrm{P}_{11}$ & Hisar Unnat & IIVR-Varanasi \\
$\mathrm{P}_{12}$ & IC -43132 & IIVR-Varanasi \\
\hline
\end{tabular}

(Table 1) and $66 \mathrm{~F}_{1}$ hybrids derived by crossing these 12 genotypes in all possible combinations in diallel fashion (excluding reciprocals) by hand emasculation and pollination during earlier spring-summer, 2012. The experiment was laid out in a Randomized Block Design (RBD) with three replications during rainy season of 2013. Each genotype was sown in 12 rows of 3 meter length accommodating 10 plants in each row. The observations were taken from randomly selected five competitive plants of parents and their crosses in each replication. The analysis of variance was used for testing as to whether there exists a significant difference between the genotypes and their hybrids. It was carried out following the statistical procedure of RBD analysis (Panse and Sukhatme, 1989) for each of the genotypes and their derived hybrids. Heterosis was calculated over superior parent (heterobeltiosis)and standard variety i.e. checks (economic heterosis), following the method described by Kempthorne (1957). The test of significance of heterosis was accomplished by the Student's ' $t$ ' test (Student, 1908a).

\section{RESULTS AND DISCUSSION}

The extent of heterobeltiosis and standard heterosis were expressed as percent increase or decrease in hybrid performance in comparison to better parent and standard check varieties, respectively. The mean performance of the genotypes $\left(\mathrm{F}_{1} \mathrm{~s}\right)$ and per cent of heterosis (over better parents and standard check) are presented in Table 2.

The heterotic performance over better-parents towards earliness (days to first flowering) was maximum in crosses $\mathrm{P}_{2} \times \mathrm{P}_{10}(-20.83 \%)$.Out of 41 crosscombinations, five best combination showed significant heterobeltiosis for days to $50 \%$ flowering viz., $\mathrm{P}_{3}$ $\times \mathrm{P}_{12}(-17.31 \%), \mathrm{P}_{2} \times \mathrm{P}_{10}(-16.67 \%), \mathrm{P}_{4} \times \mathrm{P}_{10}(-16.67$ $\%), \mathrm{P}_{2} \times \mathrm{P}_{9}(-13.21 \%)$ and $\mathrm{P}_{1} \times \mathrm{P}_{9}(-13.2 \%)$. The estimates of heterosis for plant height in hybrids varied from $-31.83 \%\left(\mathrm{P}_{3} \times \mathrm{P}_{9}\right)$ to $50.75 \%\left(\mathrm{P}_{1} \times \mathrm{P}_{4}\right)$ over better parents. The manifestation of heterosis in desirable direction for internodal length over the better parents was recorded in 20 cross-combinations. However, extent of heterobeltiosis ranged from $-37.16 \%\left(\mathrm{P}_{3} \times\right.$ $\left.\mathrm{P}_{8}\right)$ to $68.75 \%\left(\mathrm{P}_{1} \times \mathrm{P}_{2}\right)$. The heterotic increase in the number of pods per plant over their better parent was recorded in 32 hybrids and the increase varied from $0.59 \%\left(\mathrm{P}_{4} \times \mathrm{P}_{6}\right)$ to $62.82 \%\left(\mathrm{P}_{2} \times \mathrm{P}_{6}\right)$. The highest numbers of seeds per pod 54.84 were recorded in $\mathrm{P}_{8}$ and lowest 41.27 in pods of $\mathrm{P}_{5}$, while, among hybrids it was recorded from 46.27 as in $\mathrm{P}_{3} \times \mathrm{P}_{6}$ to 58.96 in $\mathrm{P}_{9} \times \mathrm{P}_{10}$. The five best parents which had high mean pod yield were $\mathrm{P}_{8}\left(106.26 \mathrm{q} \mathrm{ha}^{-1}\right), \mathrm{P}_{3}\left(101.23 \mathrm{q} \mathrm{ha}^{-1}\right), \mathrm{P}_{11}$ (93.34 q ha- $\left.{ }^{1}\right), \mathrm{P}_{4}\left(79.75 \mathrm{q} \mathrm{ha}^{-1}\right)$ and $\mathrm{P}_{6}\left(79.02 \mathrm{q} \mathrm{ha}^{-1}\right)$ whereas among hybrids, five best cross combinations were $\mathrm{P}_{2} \times \mathrm{P}_{6}\left(144.16 \mathrm{q} \mathrm{ha}^{-1}\right), \mathrm{P}_{4} \times \mathrm{P}_{7}\left(118.16 \mathrm{q} \mathrm{ha} \mathrm{a}^{-1}\right), \mathrm{P}_{1}$ $\times \mathrm{P}_{12}\left(117.78 \mathrm{q} \mathrm{ha}^{-1}\right), \mathrm{P}_{5} \times \mathrm{P}_{12}\left(115.19 \mathrm{q} \mathrm{ha}^{-1}\right)$ and $\mathrm{P}_{1} \times$ $\mathrm{P}_{3}\left(113.05 \mathrm{q} \mathrm{ha}^{-1}\right)$.

A close observation of table 2 revealed that nine cross combinations showed significant standard heterosis for days to first flowering with highest in $\mathrm{P}_{2} \times \mathrm{P}_{10}(-15.56$ $\%)$, followed by $\mathrm{P}_{5} \times \mathrm{P}_{6}(-9.89 \%)$ and $\mathrm{P}_{3} \times \mathrm{P}_{12}(-9.76$ $\%)$ whereas, seventeen cross combinations showed significant relative heterosis for earliness with best three combination were $\mathrm{P}_{2} \times \mathrm{P}_{10}(-14.56 \%), \mathrm{P}_{2} \times \mathrm{P}_{9}$

Table 2. Heterosis (\%) over better parent (BP) and the commercial check (CC) for Days to first flowering Days to $50 \%$ flowering Node at which first flower appear Plant height $(\mathrm{cm})$ Number of branches per plant in okra.

\begin{tabular}{|c|c|c|c|c|c|c|c|c|c|c|}
\hline \multirow[t]{2}{*}{ Hybrids } & \multicolumn{2}{|c|}{ Days to first flowering } & \multicolumn{2}{|c|}{ Days to $50 \%$ flowering } & \multicolumn{2}{|c|}{$\begin{array}{c}\text { Node at which first } \\
\text { flower appear }\end{array}$} & \multicolumn{2}{|c|}{ Plant height (cm) } & \multicolumn{2}{|c|}{$\begin{array}{c}\text { Number of branches } \\
\text { per plant }\end{array}$} \\
\hline & BP & $\mathbf{C C}$ & BP & $\mathbf{C C}$ & BP & $\mathrm{CC}$ & B P & $\mathbf{C C}$ & BP & $\mathrm{CC}$ \\
\hline $\mathrm{P}_{1} \times \mathrm{P}_{2}$ & 2.38 & -0.76 & $-6.49 *$ & -2.04 & 9.41 & -13.89 & $40.87 * *$ & $24.56 * *$ & $136.36 * *$ & $67.47^{*}$ \\
\hline $\mathrm{P}_{1} \times \mathrm{P}_{3}$ & 2.38 & -0.76 & $-7.69 * *$ & -2.04 & -5.26 & 0.00 & 7.58 & $43.70 * *$ & 60.71 & 44.93 \\
\hline $\mathrm{P}_{1} \times \mathrm{P}_{4}$ & $10.26 * *$ & -0.76 & $11.63 * *$ & -2.04 & 22.35 & -3.70 & $50.75 * *$ & $43.70 * *$ & $126.92 * *$ & $90.02 * *$ \\
\hline $\mathrm{P}_{1} \times \mathrm{P}_{5}$ & -4.44 & -0.76 & $-6.96 *$ & 0.00 & -1.42 & $28.70 * *$ & $-12.31 *$ & 1.64 & $74.19 *$ & $73.91^{*}$ \\
\hline $\mathrm{P}_{1} \times \mathrm{P}_{6}$ & 0.00 & 6.16 & $20.00 * *$ & $10.20 * *$ & 2.83 & 0.93 & $-15.45 * *$ & 7.19 & 59.09 & 12.72 \\
\hline $\mathrm{P}_{1} \times \mathrm{P}_{7}$ & $15.38 * *$ & 3.85 & 2.68 & 4.08 & $31.25 * *$ & $36.11 * *$ & 0.42 & 10.58 & 10.00 & 6.28 \\
\hline $\mathrm{P}_{1} \times \mathrm{P}_{8}$ & -0.77 & -0.76 & -2.04 & -2.04 & 0.93 & 0.93 & -2.84 & -2.84 & -3.23 & -3.38 \\
\hline $\mathrm{P}_{1} \times \mathrm{P}_{9}$ & -1.52 & 0.01 & $-13.21 * *$ & $-6.12 *$ & $-26.67 * *$ & -18.52 & $-15.83 * *$ & 9.77 & -40.91 & -58.13 \\
\hline $\mathrm{P}_{1} \times \mathrm{P}_{10}$ & $-12.50 * *$ & -3.07 & $-11.11 * *$ & -2.04 & -12.28 & -7.41 & $-22.19 * *$ & 7.25 & $86.36^{*}$ & 32.05 \\
\hline $\mathrm{P}_{1} \times \mathrm{P}_{11}$ & 0.79 & -2.30 & $6.67 *$ & -2.04 & $33.68 * *$ & 17.59 & $16.82 * *$ & $35.95 * *$ & 27.78 & 48.15 \\
\hline $\mathrm{P}_{1} \times \mathrm{P}_{12}$ & $-7.50 *$ & $-14.61 * *$ & -4.55 & $-14.29 * *$ & 9.62 & 5.56 & 5.36 & $34.56 * *$ & 33.33 & 15.94 \\
\hline $\mathrm{P}_{2} \times \mathrm{P}_{3}$ & -2.38 & -5.38 & $-7.69 * *$ & -2.04 & 0.00 & 5.56 & $-14.01 * *$ & $14.85 *$ & 14.29 & 3.06 \\
\hline $\mathrm{P}_{2} \times \mathrm{P}_{4}$ & -0.79 & -3.84 & $-14.29 * *$ & $-10.20 * *$ & $81.16^{* *}$ & 15.74 & $29.57 * *$ & $23.51 * *$ & $119.23 * *$ & $83.57 * *$ \\
\hline$\underline{\mathrm{P}_{2} \times \mathrm{P}_{5}}$ & -4.44 & -0.76 & $-8.86^{* *}$ & -2.04 & -5.67 & $23.15 *$ & 5.85 & $22.69 * *$ & $119.35^{* *}$ & $119.00 * *$ \\
\hline
\end{tabular}

Contd......... 
Sujit Kumar et al. / J. Appl. \& Nat. Sci. 9 (2): 774 - 779 (2017)

\begin{tabular}{|c|c|c|c|c|c|c|c|c|c|c|}
\hline$\overline{P_{2} \times P_{6}}$ & $-8.70 * *$ & -3.07 & $-6.49 *$ & -2.04 & $20.75^{*}$ & 18.52 & 2.15 & $29.51 * *$ & $244.44 * *$ & $99.68 * *$ \\
\hline $\mathrm{P}_{2} \times \mathrm{P}_{7}$ & 2.38 & -0.76 & $-10.39 * *$ & $-6.12 *$ & 5.36 & 9.26 & -4.73 & 4.91 & 13.33 & 9.50 \\
\hline $\mathrm{P}_{2} \times \mathrm{P}_{8}$ & -0.77 & -0.76 & $-6.49 *$ & -2.04 & -4.63 & -4.63 & 0.68 & 0.68 & 19.35 & 19.16 \\
\hline $\mathrm{P}_{2} \times \mathrm{P}_{9}$ & -2.27 & -0.76 & $-13.21 * *$ & $-6.12 *$ & $-28.33 * *$ & $-20.37^{*}$ & $-20.49 * *$ & 3.68 & 72.22 & -0.16 \\
\hline $\mathrm{P}_{2} \times \mathrm{P}_{10}$ & $-20.83 * *$ & $-12.30 * *$ & $-16.67 * *$ & $-8.16^{* *}$ & -10.53 & -5.56 & 1.44 & $39.82 * *$ & 31.82 & -6.60 \\
\hline $\mathrm{P}_{2} \times \mathrm{P}_{11}$ & 2.38 & -0.76 & $-8.44 * *$ & -4.08 & 1.05 & -11.11 & 11.76 & $30.06 * *$ & 8.33 & 25.60 \\
\hline $\mathrm{P}_{2} \times \mathrm{P}_{12}$ & 4.76 & 1.55 & $-6.49 *$ & -2.04 & $-25.00 *$ & $-27.78 * *$ & $-18.96 * *$ & 3.51 & -40.74 & -48.47 \\
\hline $\mathrm{P}_{3} \times \mathrm{P}_{4}$ & -2.38 & -5.38 & $-9.62 * *$ & -4.08 & $-31.58 * *$ & $-27.78 * *$ & -4.20 & $27.95 * *$ & -53.57 & -58.13 \\
\hline $\mathrm{P}_{3} \times \mathrm{P}_{5}$ & 2.22 & 6.16 & 1.90 & $9.52 * *$ & $-17.02 *$ & 8.33 & $-16.20 * *$ & 11.93 & -54.84 & -54.91 \\
\hline $\mathrm{P}_{3} \times \mathrm{P}_{6}$ & -4.35 & 1.55 & $-7.69 * *$ & -2.04 & 2.63 & 8.33 & 2.93 & $37.49 * *$ & 35.71 & 22.38 \\
\hline $\mathrm{P}_{3} \times \mathrm{P}_{7}$ & $7.94 *$ & 4.62 & -1.92 & 4.08 & 7.89 & 13.89 & -1.66 & $31.35^{* *}$ & -3.33 & -6.60 \\
\hline $\mathrm{P}_{3} \times \mathrm{P}_{8}$ & 2.31 & 2.32 & $-5.77 *$ & 0.00 & $-19.30 *$ & -14.81 & $-19.44 * *$ & 7.60 & -54.84 & -54.91 \\
\hline $\mathrm{P}_{3} \times \mathrm{P}_{9}$ & -2.27 & -0.76 & $-7.55 * *$ & 0.00 & -11.67 & -1.85 & $-31.83 * *$ & -8.95 & $-82.14 *$ & $-83.90 * *$ \\
\hline $\mathrm{P}_{3} \times \mathrm{P}_{10}$ & -5.56 & 4.62 & $-5.56^{*}$ & 4.08 & $31.58 * *$ & $38.89 * *$ & $19.56^{* *}$ & $64.80 * *$ & $139.29 * *$ & $115.78 * *$ \\
\hline $\mathrm{P}_{3} \times \mathrm{P}_{11}$ & -4.76 & $-7.69 *$ & $-9.62 * *$ & -4.08 & 0.00 & 5.56 & $17.16^{* *}$ & $56.49 * *$ & 13.89 & 32.05 \\
\hline $\mathrm{P}_{3} \times \mathrm{P}_{12}$ & $-11.90 * *$ & $-14.61 * *$ & $-17.31 * *$ & $-12.24 * *$ & -2.63 & 2.78 & -6.48 & $24.91 * *$ & 35.71 & 22.38 \\
\hline $\mathrm{P}_{4} \times \mathrm{P}_{5}$ & $-11.11 * *$ & $-7.69 *$ & $-10.76^{* *}$ & -4.08 & $-36.88 * *$ & -17.59 & $-16.25 * *$ & -2.92 & 16.13 & 15.94 \\
\hline $\mathrm{P}_{4} \times \mathrm{P}_{6}$ & -2.17 & 3.85 & $11.11 * *$ & 2.04 & $21.70^{*}$ & $19.44 *$ & 4.75 & $32.81 * *$ & 57.69 & 32.05 \\
\hline $\mathrm{P}_{4} \times \mathrm{P}_{7}$ & $10.26 * *$ & -0.76 & $-11.41 * *$ & $-10.20 * *$ & -3.57 & 0.00 & $13.33^{*}$ & $24.80 * *$ & 13.33 & 9.50 \\
\hline $\mathrm{P}_{4} \times \mathrm{P}_{8}$ & -0.77 & -0.76 & -2.04 & -2.04 & -0.93 & -0.93 & $16.08^{*}$ & $16.08 *$ & 9.68 & 9.50 \\
\hline $\mathrm{P}_{4} \times \mathrm{P}_{9}$ & -2.27 & -0.76 & $-8.81 * *$ & -1.36 & -12.50 & -2.78 & $-15.52 * *$ & 10.18 & -50.00 & -58.13 \\
\hline $\mathrm{P}_{4} \times \mathrm{P}_{10}$ & $-12.50 * *$ & -3.07 & $-16.67 * *$ & $-8.16 * *$ & -2.63 & 2.78 & -1.99 & $35.09 * *$ & -11.54 & -25.93 \\
\hline $\mathrm{P}_{4} \times \mathrm{P}_{11}$ & -4.76 & $-7.69 *$ & 0.00 & $-8.16^{* *}$ & -3.16 & -14.81 & -9.35 & 5.50 & $-88.89 * *$ & $-87.12 * *$ \\
\hline $\mathrm{P}_{4} \times \mathrm{P}_{12}$ & -5.00 & $-12.30 * *$ & $-6.82 *$ & $-16.33 * *$ & -4.81 & -8.33 & -9.89 & $15.09 *$ & -48.15 & -54.91 \\
\hline $\mathrm{P}_{5} \times \mathrm{P}_{6}$ & $-10.87 * *$ & -5.38 & -1.27 & $6.12 *$ & 8.51 & $41.67 * *$ & 2.86 & $30.41 * *$ & 54.84 & 54.59 \\
\hline $\mathrm{P}_{5} \times \mathrm{P}_{7}$ & -2.22 & 1.55 & 0.00 & $7.48^{*}$ & -11.35 & 15.74 & $-15.49 * *$ & -2.05 & 19.35 & 19.16 \\
\hline $\mathrm{P}_{5} \times \mathrm{P}_{8}$ & -4.44 & -0.76 & $-7.59 * *$ & -0.68 & -1.42 & $28.70 * *$ & 8.27 & 25.50 & $122.58 * *$ & $122.22 * *$ \\
\hline $\mathrm{P}_{5} \times \mathrm{P}_{9}$ & -4.44 & -0.76 & $-6.29 *$ & 1.36 & -2.84 & $26.85 * *$ & -8.88 & $18.83 * *$ & $70.97 *$ & $70.69 *$ \\
\hline $\mathrm{P}_{5} \times \mathrm{P}_{10}$ & $-10.42 * *$ & -0.76 & $-8.02 * *$ & 1.36 & -3.55 & $25.93 * *$ & -8.40 & $26.26 * *$ & $112.90 * *$ & $112.56^{* *}$ \\
\hline $\mathrm{P}_{5} \times \mathrm{P}_{11}$ & -5.93 & -2.30 & $-12.66^{* *}$ & $-6.12 *$ & -14.18 & 12.04 & 5.63 & $22.92 * *$ & 36.11 & 57.81 \\
\hline $\mathrm{P}_{5} \times \mathrm{P}_{12}$ & 0.00 & 3.85 & -3.80 & 3.40 & 11.35 & $45.37 * *$ & 9.89 & $40.35^{* *}$ & $112.90 * *$ & $112.56^{* *}$ \\
\hline $\mathrm{P}_{6} \times \mathrm{P}_{7}$ & $-12.32 * *$ & $-6.92 *$ & -1.34 & 0.00 & 16.07 & $20.37 *$ & $16.97 * *$ & $48.30 * *$ & $83.33 * *$ & $77.13^{*}$ \\
\hline $\mathrm{P}_{6} \times \mathrm{P}_{8}$ & $-6.52 *$ & -0.76 & $6.12 *$ & $6.12 *$ & 15.74 & 15.74 & -5.35 & $20.00 * *$ & 32.26 & 32.05 \\
\hline $\mathrm{P}_{6} \times \mathrm{P}_{9}$ & $-8.70 * *$ & -3.07 & $-7.55 * *$ & 0.00 & -1.67 & 9.26 & 4.84 & $36.73 * *$ & $138.89 * *$ & 38.49 \\
\hline $\mathrm{P}_{6} \times \mathrm{P}_{10}$ & $-10.42 * *$ & -0.76 & $-12.96 * *$ & -4.08 & $23.68 * *$ & $30.56 * *$ & 7.76 & $48.54 * *$ & $150.00 * *$ & $77.13 *$ \\
\hline $\mathrm{P}_{6} \times \mathrm{P}_{11}$ & $-10.87 * *$ & -5.38 & 0.00 & -8.16 & $31.13 * *$ & $28.70 * *$ & 10.33 & $39.88 * *$ & $102.78 * *$ & $135.10^{* *}$ \\
\hline $\mathrm{P}_{6} \times \mathrm{P}_{12}$ & $-15.22 * *$ & $-9.99 * *$ & $-6.67 *$ & -14.29 & $24.53 *$ & $22.22 *$ & 10.62 & $41.29 * *$ & $81.48 *$ & 57.81 \\
\hline $\mathrm{P}_{7} \times \mathrm{P}_{8}$ & -5.38 & -5.38 & $-9.40 * *$ & -8.16 & 8.04 & 12.04 & 2.92 & 13.33 & 58.06 & 57.81 \\
\hline $\mathrm{P}_{7} \times \mathrm{P}_{9}$ & -4.55 & -3.07 & $-7.55 * *$ & 0.00 & -6.67 & 3.70 & $-24.39 * *$ & -1.40 & -36.67 & -38.81 \\
\hline $\mathrm{P}_{7} \times \mathrm{P}_{10}$ & 0.00 & $10.78 * *$ & -0.62 & 9.52 & 4.39 & 10.19 & $-15.74 * *$ & $16.14 *$ & -36.67 & -38.81 \\
\hline $\mathrm{P}_{7} \times \mathrm{P}_{11}$ & 0.00 & -3.07 & -4.03 & -2.72 & -13.39 & -10.19 & $-12.66^{*}$ & 1.64 & -25.00 & -13.04 \\
\hline $\mathrm{P}_{7} \times \mathrm{P}_{12}$ & 5.00 & -3.07 & -1.34 & 0.00 & -14.29 & -11.11 & $-18.96 * *$ & 3.51 & 3.33 & -0.16 \\
\hline $\mathrm{P}_{8} \times \mathrm{P}_{9}$ & -2.27 & -0.76 & $-7.55 * *$ & 0.00 & $-21.67 *$ & -12.96 & $-29.60 * *$ & -8.19 & 0.00 & -0.16 \\
\hline $\mathrm{P}_{8} \times \mathrm{P}_{10}$ & -2.08 & $8.47 *$ & -2.47 & 7.48 & -14.91 & -10.19 & $-25.16^{* *}$ & 3.16 & 12.90 & 12.72 \\
\hline $\mathrm{P}_{8} \times \mathrm{P}_{11}$ & 1.54 & 1.55 & 0.00 & 0.00 & $-24.07 *$ & $-24.07 *$ & $-28.24 * *$ & $-16.49^{*}$ & -2.78 & 12.72 \\
\hline $\mathrm{P}_{8} \times \mathrm{P}_{12}$ & -3.08 & -3.07 & -2.04 & -2.04 & -15.74 & -15.74 & $-22.25 * *$ & -0.70 & 45.16 & 44.93 \\
\hline $\mathrm{P}_{9} \times \mathrm{P}_{10}$ & $-12.50 * *$ & -3.07 & $-11.11 * *$ & -2.04 & $-18.33^{*}$ & -9.26 & $-17.88 * *$ & 13.19 & $90.91 *$ & 35.27 \\
\hline $\mathrm{P}_{9} \times \mathrm{P}_{11}$ & -2.27 & -0.76 & -1.26 & 6.80 & $-19.17 *$ & -10.19 & $-20.36 * *$ & 3.86 & -36.11 & -25.93 \\
\hline $\mathrm{P}_{9} \times \mathrm{P}_{12}$ & -2.27 & -0.76 & $-5.66^{*}$ & 2.04 & -10.83 & -0.93 & -8.07 & $19.88^{* *}$ & -25.93 & -35.59 \\
\hline $\mathrm{P}_{10} \times \mathrm{P}_{11}$ & -2.08 & $8.47^{*}$ & -1.85 & 8.16 & -16.67 & -12.04 & $-15.06 * *$ & $17.08 *$ & 33.33 & 54.59 \\
\hline $\mathrm{P}_{10} \times \mathrm{P}_{12}$ & $-14.58 * *$ & -5.38 & $-11.11 * *$ & -2.04 & -16.67 & -12.04 & -0.13 & $37.66 * *$ & $122.22 * *$ & $93.24 * *$ \\
\hline $\mathrm{P}_{11} \times \mathrm{P}_{12}$ & 0.00 & -3.07 & 2.22 & -6.12 & -3.85 & -7.41 & -1.69 & $25.56^{* *}$ & -38.89 & -29.15 \\
\hline S.E.D. & 1.497 & 1.497 & 1.414 & 1.414 & 0.689 & .689 & 3.917 & 3.917 & 0.624 & 0.624 \\
\hline C.D. at $5 \%$ & 2.990 & 2.990 & 2.825 & 2.825 & 1.375 & 1.375 & 7.822 & 7.822 & 1.246 & 1.246 \\
\hline C.D. at $1 \%$ & 3.905 & 3.905 & 3.689 & 3.689 & 1.796 & 1.796 & 10.215 & 10.215 & 1.627 & 1.267 \\
\hline
\end{tabular}

** Significant at $5 \%$ and * significant at $1 \%$ probability level. BP and CC - Heterosis over better parent and commercial check (Kashi Pragati) respectively.

Table 2. Heterosis (\%) over better parent (BP) and the commercial check (CC) for Internodal distance (cm) Number of pods per plant Pod weight (g) Number of seeds per pod Pod yield $(q /$ ha) in okra.

\begin{tabular}{|c|c|c|c|c|c|c|c|c|c|c|}
\hline \multirow{2}{*}{ Hybrids } & \multicolumn{2}{|c|}{ Internodal distance $(\mathrm{cm})$} & \multicolumn{2}{|c|}{ Number of pods per plant } & \multicolumn{2}{|c|}{ Pod weight (g) } & \multicolumn{2}{|c|}{ Number of seeds per pod } & \multicolumn{2}{|c|}{ Pod yield (q/ ha) } \\
\hline & BP & $\mathrm{CC}$ & $\mathbf{B P}$ & $\mathrm{CC}$ & BP & $\mathrm{CC}$ & B P & $\mathrm{CC}$ & BP & $\mathrm{CC}$ \\
\hline$\overline{P_{1} \times P_{2}}$ & $68.75 * *$ & -1.75 & 7.69 & -1.75 & $28.46^{* *}$ & -7.80 & 11.94* & -2.07 & $39.69 * *$ & -9.24 \\
\hline $\mathrm{P}_{1} \times \mathrm{P}_{3}$ & -10.81 & $15.47 *$ & 4.47 & $15.47 *$ & 6.92 & -7.60 & $14.43 *$ & -2.68 & 11.68 & 6.39 \\
\hline $\mathrm{P}_{1} \times \mathrm{P}_{4}$ & -1.20 & 7.60 & 8.88 & 7.60 & 12.96 & $-14.45^{*}$ & 6.60 & -7.78 & $22.85 *$ & -7.80 \\
\hline $\mathrm{P}_{1} \times \mathrm{P}_{5}$ & $-34.15 * *$ & -12.87 & 16.41 & -12.87 & 7.76 & $-16.98 *$ & 7.86 & -8.27 & $26.85 * *$ & $-27.88 * *$ \\
\hline $\mathrm{P}_{1} \times \mathrm{P}_{6}$ & $-26.24 * *$ & $-26.32 * *$ & -16.56 & $-26.32 * *$ & 1.79 & $-14.29 *$ & 10.20 & -1.59 & $-15.58 *$ & $-37.22 * *$ \\
\hline $\mathrm{P}_{1} \times \mathrm{P}_{7}$ & $-35.81 * *$ & $-16.96 *$ & 2.16 & $-16.96^{*}$ & $22.52 *$ & -12.55 & -3.25 & -5.96 & $29.94 * *$ & $-27.50 * *$ \\
\hline
\end{tabular}

Contd.......... 
Sujit Kumar et al. / J. Appl. \& Nat. Sci. 9 (2): 774 - 779 (2017)

\begin{tabular}{|c|c|c|c|c|c|c|c|c|c|c|}
\hline$\overline{\mathrm{P}_{1} \times \mathrm{P}_{8}}$ & -9.81 & -10.53 & -10.53 & -10.53 & $-23.65 * *$ & $-23.63 * *$ & -0.61 & -0.61 & $-32.77 * *$ & $-32.77 * *$ \\
\hline $\mathrm{P}_{1} \times \mathrm{P}_{9}$ & -8.03 & $-21.05 * *$ & -2.17 & $-21.05^{* *}$ & -11.78 & $-28.82 * *$ & 2.74 & -4.26 & -13.84 & $-43.72 * *$ \\
\hline $\mathrm{P}_{1} \times \mathrm{P}_{10}$ & 4.25 & 11.70 & $49.22 * *$ & 11.70 & 4.60 & $-25.34 * *$ & -7.88 & $-13.37 *$ & $55.66 * *$ & $-16.80 * *$ \\
\hline $\mathrm{P}_{1} \times \mathrm{P}_{11}$ & 17.86 & 14.62 & 10.73 & 14.62 & -2.45 & $-16.51^{*}$ & $11.26^{*}$ & 3.27 & 8.51 & -4.68 \\
\hline $\mathrm{P}_{1} \times \mathrm{P}_{12}$ & $-21.48 *$ & $26.32 * *$ & $40.26 * *$ & $26.32 * *$ & 11.48 & -12.35 & 1.92 & -9.85 & $56.67 * *$ & 10.82 \\
\hline $\mathrm{P}_{2} \times \mathrm{P}_{3}$ & $-33.65 * *$ & 2.92 & -6.88 & 2.92 & 8.93 & -5.86 & 4.44 & -8.63 & 1.85 & -2.97 \\
\hline $\mathrm{P}_{2} \times \mathrm{P}_{4}$ & -1.72 & -11.70 & -10.65 & -11.70 & 8.57 & $-17.78 *$ & 8.47 & -5.11 & -2.98 & $-27.19 * *$ \\
\hline $\mathrm{P}_{2} \times \mathrm{P}_{5}$ & $-22.11 *$ & 1.75 & 11.54 & 1.75 & $-18.81 *$ & $-37.45 * *$ & 4.03 & -9.00 & -2.90 & $-36.91 * *$ \\
\hline $\mathrm{P}_{2} \times \mathrm{P}_{6}$ & $-29.65 * *$ & $48.54 * *$ & $62.82 * *$ & $48.54 * *$ & 8.37 & -8.75 & 5.17 & -6.08 & $82.42 * *$ & $35.66^{* *}$ \\
\hline $\mathrm{P}_{2} \times \mathrm{P}_{7}$ & $-26.94 * *$ & 10.53 & $21.15^{*}$ & 10.53 & 11.80 & $-19.75^{* *}$ & 4.63 & 1.69 & $37.21 * *$ & -10.85 \\
\hline $\mathrm{P}_{2} \times \mathrm{P}_{8}$ & -8.70 & 8.77 & 8.77 & 8.77 & $-25.63 * *$ & $-25.61 * *$ & -4.50 & -4.50 & $-19.39 *$ & $-19.39 * *$ \\
\hline $\mathrm{P}_{2} \times \mathrm{P}_{9}$ & -7.86 & 6.43 & $16.67 *$ & 6.43 & -14.03 & $-30.64 * *$ & 0.39 & -6.45 & 13.54 & $-25.84 * *$ \\
\hline $\mathrm{P}_{2} \times \mathrm{P}_{10}$ & $20.47 *$ & $22.22 * *$ & $33.97 * *$ & $22.22 * *$ & 8.94 & $-21.81 * *$ & -3.75 & -9.48 & $47.40 * *$ & -4.23 \\
\hline $\mathrm{P}_{2} \times \mathrm{P}_{11}$ & $21.61 *$ & 14.04 & 10.17 & 14.04 & -6.06 & $-19.60 * *$ & -3.14 & -10.09 & 4.69 & -8.04 \\
\hline $\mathrm{P}_{2} \times \mathrm{P}_{12}$ & $-20.00 *$ & -2.34 & 7.05 & -2.34 & -5.04 & $-25.34 * *$ & 3.30 & -8.63 & 1.22 & $-28.41 * *$ \\
\hline $\mathrm{P}_{3} \times \mathrm{P}_{4}$ & 1.35 & 12.28 & 1.59 & 12.28 & $-16.40 *$ & $-27.75 * *$ & 8.29 & -6.32 & $-14.23^{*}$ & $-18.29 * *$ \\
\hline $\mathrm{P}_{3} \times \mathrm{P}_{5}$ & -14.86 & $-16.96 *$ & $-24.87 * *$ & $-16.96^{*}$ & $-16.63 *$ & $-27.95 * *$ & $18.81 * *$ & -10.21 & $-37.11 * *$ & $-40.09 * *$ \\
\hline $\mathrm{P}_{3} \times \mathrm{P}_{6}$ & $-27.97 * *$ & $-20.47 * *$ & $-28.04 * *$ & $-20.47 * *$ & -15.03 & $-26.56 * *$ & -5.58 & $-15.68 * *$ & $-38.73 * *$ & $-41.63 * *$ \\
\hline $\mathrm{P}_{3} \times \mathrm{P}_{7}$ & $-26.35 * *$ & -1.17 & -10.58 & -1.17 & $-19.79 *$ & $-30.68 * *$ & 0.63 & -2.19 & $-28.05 * *$ & $-31.46^{* *}$ \\
\hline $\mathrm{P}_{3} \times \mathrm{P}_{8}$ & $-37.16 * *$ & $-19.88 * *$ & $-27.51 * *$ & $-19.88 * *$ & $-16.76^{*}$ & $-16.75^{*}$ & -3.04 & -3.04 & $-33.48 * *$ & $-33.48 * *$ \\
\hline $\mathrm{P}_{3} \times \mathrm{P}_{9}$ & $-27.03 * *$ & $-45.03 * *$ & $-50.26 * *$ & $-45.03 * *$ & -13.06 & $-24.86 * *$ & -0.78 & -7.54 & $-56.85 * *$ & $-58.90 * *$ \\
\hline $\mathrm{P}_{3} \times \mathrm{P}_{10}$ & $-15.54 *$ & -11.70 & $-20.11 * *$ & -11.70 & $-17.20 *$ & $-28.44 * *$ & -4.65 & $-10.33 *$ & $-34.10 * *$ & $-37.23 * *$ \\
\hline $\mathrm{P}_{3} \times \mathrm{P}_{11}$ & $-20.54 * *$ & 9.94 & -0.53 & 9.94 & -16.22 & $-27.59 * *$ & 0.65 & -6.57 & $-16.26^{*}$ & $-20.23 * *$ \\
\hline $\mathrm{P}_{3} \times \mathrm{P}_{12}$ & $-36.49 * *$ & $18.71^{*}$ & 7.41 & $18.71 *$ & -15.39 & $-26.88 * *$ & 5.91 & -6.32 & -9.53 & $-13.82 *$ \\
\hline $\mathrm{P}_{4} \times \mathrm{P}_{5}$ & $-28.62 * *$ & -3.51 & -2.37 & -3.51 & -5.09 & $-26.88 * *$ & -1.26 & $-14.59 * *$ & -6.36 & $-29.73 * *$ \\
\hline $\mathrm{P}_{4} \times \mathrm{P}_{6}$ & -15.89 & -0.58 & 0.59 & -0.58 & -1.93 & $-17.42 *$ & 10.88 & -0.98 & 8.36 & $-18.68 * *$ \\
\hline $\mathrm{P}_{4} \times \mathrm{P}_{7}$ & -0.48 & $25.15^{* *}$ & $26.63 * *$ & $25.15 * *$ & $18.92 *$ & -9.94 & -7.50 & -10.09 & $48.32 * *$ & 11.31 \\
\hline $\mathrm{P}_{4} \times \mathrm{P}_{8}$ & 1.72 & 1.75 & 1.75 & 1.75 & -12.37 & -12.35 & -6.08 & -6.08 & -10.85 & -10.86 \\
\hline $\mathrm{P}_{4} \times \mathrm{P}_{9}$ & -3.42 & $-15.79 *$ & -14.79 & $-15.79 *$ & 1.82 & $-17.85^{*}$ & 0.26 & -6.57 & -7.49 & $-30.57 * *$ \\
\hline $\mathrm{P}_{4} \times \mathrm{P}_{10}$ & -1.57 & 3.51 & 4.73 & 3.51 & 7.37 & $-18.69 * *$ & -6.72 & $-12.28^{*}$ & 11.44 & $-16.37 * *$ \\
\hline $\mathrm{P}_{4} \times \mathrm{P}_{11}$ & 6.35 & $-19.30 *$ & $-22.03 * *$ & $-19.30 *$ & -7.63 & $-20.94 * *$ & -9.03 & $-15.56 * *$ & $-27.28 * *$ & $-36.12 * *$ \\
\hline $\mathrm{P}_{4} \times \mathrm{P}_{12}$ & -14.07 & $-21.64 * *$ & $-20.71 * *$ & $-21.64 * *$ & 8.26 & $-14.89 *$ & 0.69 & $-10.94 *$ & -11.86 & $-33.85 * *$ \\
\hline $\mathrm{P}_{5} \times \mathrm{P}_{6}$ & -3.55 & -7.60 & 4.64 & -7.60 & -2.73 & $-18.09 *$ & 2.86 & -8.15 & 0.94 & $-24.93 * *$ \\
\hline $\mathrm{P}_{5} \times \mathrm{P}_{7}$ & -12.10 & $-25.15^{* *}$ & -7.91 & $-25.15^{* *}$ & $-25.64 * *$ & $-42.72 * *$ & $-19.63 * *$ & $-21.88 * *$ & $-24.12 *$ & $-56.86 * *$ \\
\hline $\mathrm{P}_{5} \times \mathrm{P}_{8}$ & 0.98 & 8.77 & 8.77 & 8.77 & $-17.32 *$ & $-17.30^{*}$ & -2.67 & -2.68 & -12.29 & $-12.29 *$ \\
\hline $\mathrm{P}_{5} \times \mathrm{P}_{9}$ & -0.81 & -4.09 & $18.84^{*}$ & -4.09 & -13.89 & $-30.52 * *$ & 0.00 & -6.81 & 1.88 & $-33.45^{* *}$ \\
\hline $\mathrm{P}_{5} \times \mathrm{P}_{10}$ & -1.57 & 4.09 & $41.27 * *$ & 4.09 & -15.88 & $-35.19 * *$ & -7.75 & $-13.25^{*}$ & $18.25^{*}$ & $-32.77 * *$ \\
\hline $\mathrm{P}_{5} \times \mathrm{P}_{11}$ & 1.63 & $31.58 * *$ & $27.12 * *$ & $31.58 * *$ & $-16.74 *$ & $-28.74 * *$ & -7.33 & $-13.98 * *$ & 4.12 & -8.53 \\
\hline $\mathrm{P}_{5} \times \mathrm{P}_{12}$ & 11.11 & $27.49 * *$ & $41.56 * *$ & $27.49 * *$ & 8.41 & $-14.77 *$ & 6.04 & -6.20 & $53.25 * *$ & 8.40 \\
\hline $\mathrm{P}_{6} \times \mathrm{P}_{7}$ & 4.26 & -0.58 & 12.58 & -0.58 & -7.95 & $-22.49 * *$ & 0.62 & -2.19 & 3.43 & $-23.08 * *$ \\
\hline $\mathrm{P}_{6} \times \mathrm{P}_{8}$ & $-16.45^{*}$ & $-23.39 * *$ & $-23.39 * *$ & $-23.39 * *$ & $-15.46^{*}$ & $-15.44 *$ & -6.08 & -6.08 & $-35.54 * *$ & $-35.54 * *$ \\
\hline $\mathrm{P}_{6} \times \mathrm{P}_{9}$ & -9.22 & -9.94 & 1.99 & -9.94 & 11.61 & -6.02 & 5.48 & -1.71 & 13.93 & $-15.27 *$ \\
\hline $\mathrm{P}_{6} \times \mathrm{P}_{10}$ & -9.93 & 2.92 & 16.56 & 2.92 & 1.13 & $-14.85^{*}$ & 5.68 & -0.61 & $18.02 *$ & $-12.23 *$ \\
\hline $\mathrm{P}_{6} \times \mathrm{P}_{11}$ & -9.93 & $25.15^{* *}$ & $20.90 * *$ & $25.15 * *$ & -12.81 & $-25.38 * *$ & 3.53 & -3.89 & 6.67 & -6.29 \\
\hline $\mathrm{P}_{6} \times \mathrm{P}_{12}$ & -10.07 & $22.22 * *$ & $35.71 * *$ & $22.22 * *$ & -14.25 & $-27.79 * *$ & 3.67 & -7.42 & $18.83 *$ & -11.63 \\
\hline $\mathrm{P}_{7} \times \mathrm{P}_{8}$ & -0.81 & -10.53 & -10.53 & -10.53 & $-18.86^{* *}$ & $-18.84 * *$ & 5.47 & 5.46 & $-27.95 * *$ & $-27.95 * *$ \\
\hline $\mathrm{P}_{7} \times \mathrm{P}_{9}$ & 0.00 & -2.92 & $19.42 *$ & -2.92 & -14.03 & $-30.64 * *$ & -5.37 & -8.03 & 2.74 & $-32.89 * *$ \\
\hline $\mathrm{P}_{7} \times \mathrm{P}_{10}$ & -5.51 & $-28.65 * *$ & -12.23 & $-28.65 * *$ & -11.36 & $-38.52 * *$ & $-16.00 * *$ & $-18.35 * *$ & $-21.99 *$ & $-56.47 * *$ \\
\hline $\mathrm{P}_{7} \times \mathrm{P}_{11}$ & 2.42 & $-30.41 * *$ & $-32.77 * *$ & $-30.41 * *$ & -13.55 & $-26.01 * *$ & 5.38 & 2.42 & $-42.34 * *$ & $-49.35 * *$ \\
\hline $\mathrm{P}_{7} \times \mathrm{P}_{12}$ & -5.93 & $-26.32 * *$ & $-18.18^{*}$ & $-26.32 * *$ & $-20.09 *$ & $-37.17 * *$ & -10.00 & $-12.52 *$ & $-34.43 * *$ & $-53.62 * *$ \\
\hline $\mathrm{P}_{8} \times \mathrm{P}_{9}$ & 8.72 & $-26.32 * *$ & $-26.32 * *$ & -26.32 & $-20.05 * *$ & $-20.03 * *$ & -1.46 & -1.46 & $-41.56 * *$ & $-41.56 * *$ \\
\hline $\mathrm{P}_{8} \times \mathrm{P}_{10}$ & -3.15 & -4.09 & -4.09 & -4.09 & $-31.13 * *$ & $-31.12 * *$ & $-14.22 * *$ & $-14.22 * *$ & $-34.59 * *$ & $-34.59 * *$ \\
\hline $\mathrm{P}_{8} \times \mathrm{P}_{11}$ & -13.39 & -0.58 & -3.95 & -0.58 & $-18.54 * *$ & $-18.53 * *$ & -8.75 & -8.75 & $-19.35^{*}$ & $-19.35 * *$ \\
\hline $\mathrm{P}_{8} \times \mathrm{P}_{12}$ & $-17.04 *$ & 1.17 & 1.17 & 1.17 & 5.96 & 5.98 & -3.16 & -3.17 & 7.13 & 7.13 \\
\hline $\mathrm{P}_{9} \times \mathrm{P}_{10}$ & -5.51 & -14.62 & 5.80 & -14.62 & $19.43^{*}$ & -3.64 & $13.82 *$ & 7.04 & $25.94 * *$ & $-17.74 * *$ \\
\hline $\mathrm{P}_{9} \times \mathrm{P}_{11}$ & $-19.83 *$ & -14.04 & $-16.95 *$ & -14.04 & 8.83 & -6.85 & -4.04 & $-10.58 *$ & -9.14 & $-20.19 * *$ \\
\hline $\mathrm{P}_{9} \times \mathrm{P}_{12}$ & -7.41 & 6.43 & $18.18^{*}$ & 6.43 & -3.29 & $-21.97 * *$ & -6.91 & $-13.25 *$ & $16.19 *$ & $-17.81 * *$ \\
\hline $\mathrm{P}_{10} \times \mathrm{P}_{11}$ & -14.17 & 8.77 & 5.08 & 8.77 & -13.32 & $-25.81 * *$ & -0.13 & -6.08 & -8.75 & $-19.84 * *$ \\
\hline $\mathrm{P}_{10} \times \mathrm{P}_{12}$ & 0.00 & -11.11 & -1.30 & -11.11 & 1.66 & $-20.07 * *$ & 4.78 & -1.46 & -0.88 & $-29.89 * *$ \\
\hline $\mathrm{P}_{11} \times \mathrm{P}_{12}$ & -9.04 & $15.79 *$ & 11.86 & $15.79 *$ & -9.34 & $-22.41 * *$ & 8.51 & 0.72 & 2.23 & -10.20 \\
\hline S.E.D. & 0.376 & 0.376 & 0.865 & 0.865 & 1.187 & 1.187 & 2.867 & 2.867 & 6.566 & 6.566 \\
\hline C.D. at $5 \%$ & 0.752 & 0.752 & 1.727 & 1.727 & 2.370 & 2.370 & 5.725 & 5.725 & 13.114 & 13.114 \\
\hline C.D. at $1 \%$ & 0.982 & 0.982 & 2.255 & 2.255 & 3.095 & 3.095 & 7.477 & 7.477 & 17.125 & 17.125 \\
\hline
\end{tabular}

** Significant at $5 \%$ and * significant at $1 \%$ probability level. BP and CC - Heterosis over better parent and commercial check (Kashi Pragati) respectively. 
(-11.82\%), and $\mathrm{P}_{3} \times \mathrm{P}_{12}(-10.42 \%)$. Among $66 \mathrm{~F}_{1}$ hybrids, 16 combinations manifested significant standard heterosis over check 1 (VRO-5) while six combinations exhibited significant standard heterosisfor day $50 \%$ flowering with maximum in $\mathrm{P}_{4} \times \mathrm{P}_{12}(-8.89 \%)$ followed by $\mathrm{P}_{1} \times \mathrm{P}_{12}(-6.67 \%)$ and $\mathrm{P}_{6} \times \mathrm{P}_{12}(-6.67 \%)$. Perusal of heterosis data manifested four cross combinations in desired direction with maximum in $\mathrm{P}_{8} \times \mathrm{P}_{11}$ $(-19.21 \%)$ followed by $\mathrm{P}_{8} \times \mathrm{P}_{9}(-17.54 \%)$, and $\mathrm{P}_{3} \times \mathrm{P}_{8}$ $(17.12 \%)$. Eleven cross combinations for node at which first flower appear showed significant negative heterobeltiosis with best three were $\mathrm{P}_{4} \times \mathrm{P}_{5}(-36.88 \%)$ followed by $\mathrm{P}_{3} \times \mathrm{P}_{4}(-31.58 \%)$ and $\mathrm{P}_{2} \times \mathrm{P}_{9}(-28.33 \%)$ and four hybrids showed significant standard heterosis over check 1with maximum in $\mathrm{P}_{2} \times \mathrm{P}_{12}(-27.78 \%), \mathrm{P}_{3} \times$ $\mathrm{P}_{4}(-27.78 \%)$ followed by $\mathrm{P}_{8} \times \mathrm{P}_{4}(-24.07 \%)$ and $\mathrm{P}_{2} \times$ $\mathrm{P}_{9}(-0.37 \%)$. Out of 66,22 cross combination showed significant mid parental heterosis with best three combination viz. $\mathrm{P}_{1} \times \mathrm{P}_{4}(56.41 \%), \mathrm{P}_{1} \times \mathrm{P}_{2}(49.16 \%)$ and $\mathrm{P}_{2}$ $\times \mathrm{P}_{4}(42.03 \%)$ for plant height while all combination showed significant standard heterosis over check 1 . Out of 66, 27 cross combination showed significant average heterosis for number of branches with best three were $\mathrm{P}_{2} \times \mathrm{P}_{6}(254.29 \%), \mathrm{P}_{2} \times \mathrm{P}_{5}(183.33 \%), \mathrm{P}_{6} \times$ $\mathrm{P}_{10}(175 \%)$ while three best significant heterobeltiosis recorded in cross combination $\mathrm{P}_{2} \times \mathrm{P}_{6}(244.44 \%), \mathrm{P}_{6} \times$ $\mathrm{P}_{10}(150 \%)$ and $\mathrm{P}_{3} \times \mathrm{P}_{10}(139.29 \%)$.

Extent of average heterosis for internodal distance were ranged from $-33.57 \%$ to $70.53 \%$ out of which, 11 hybrids showed significant negative mid parental heterosis $\mathrm{P}_{3} \times \mathrm{P}_{12}(-33.57 \%)$ followed by $\mathrm{P}_{1} \times \mathrm{P}_{5}$ $(-27.64 \%), \mathrm{P}_{4} \times \mathrm{P}_{5}(-26.71 \%)$ while, $\mathrm{P}_{3} \times \mathrm{P}_{9}(-45.03$ $\%), \mathrm{P}_{7} \times \mathrm{P}_{11}(-30.41 \%)$ and $\mathrm{P}_{7} \times \mathrm{P}_{10}(-28.65 \%)$ showed significant standard heterosis over check 1 . For no of pods per plant, 15 hybrids showed significant heterobeltiosis with three best were $\mathrm{P}_{2} \times \mathrm{P}_{6}(62.82 \%)$, $\mathrm{P}_{1} \times \mathrm{P}_{10}(49.22 \%)$ and $\mathrm{P}_{5} \times \mathrm{P}_{12}(41.56 \%)$ whereas 11 cross combinations with maximum value, $\mathrm{P}_{2} \times \mathrm{P}_{6}$ $(48.54 \%)$ followed by $\mathrm{P}_{5} \times \mathrm{P}_{11}(31.58 \%)$ and $\mathrm{P}_{5} \times \mathrm{P}_{12}$ $(27.49 \%)$, standard heterosis over check 1 and $\mathrm{P}_{2} \times \mathrm{P}_{9}$ $(43.50 \%), \mathrm{P}_{5} \times \mathrm{P}_{11}(27.12 \%)$ and $\mathrm{P}_{5} \times \mathrm{P}_{12}(23.16 \%)$ over standard check 2 (Hissar Unnat) for same trait. For pod yield, 24 hybrids manifested significant average heterosis with best three cross combinations were $\mathrm{P}_{2} \times \mathrm{P}_{10}(102.08 \%), \mathrm{P}_{1} \times \mathrm{P}_{10}(99.85 \%)$ and $\mathrm{P}_{2} \times \mathrm{P}_{6}$ $(94.72 \%)$ where as $\mathrm{P}_{2} \times \mathrm{P}_{6}(82.42 \%), \mathrm{P}_{10} \times \mathrm{P}_{12}(56.66$ $\%), \mathrm{P}_{1} \times \mathrm{P}_{10}(56.66 \%)$ and $\mathrm{P}_{5} \times \mathrm{P}_{12}(53.25 \%)$ recorded significant heterobeltiosis, five combinations namely $\mathrm{P}_{2}$ $\times \mathrm{P}_{6}(54.44 \%), \mathrm{P}_{4} \times \mathrm{P}_{7}(26.72 \%), \mathrm{P}_{1} \times \mathrm{P}_{12}(26.16 \%)$, $\mathrm{P}_{5} \times \mathrm{P}_{12}(23.41 \%), \mathrm{P}_{8} \times \mathrm{P}_{12}(21.96 \%)$ and $\mathrm{P}_{1} \times \mathrm{P}_{3}$ $(21.12 \%)$ showed significant standard heterosis over check 2.

The best common crosses based on per se performance as well as heterosis over better parent and standard check, the crosses found promising were $\mathrm{P}_{1} \times \mathrm{P}_{4}, \mathrm{P}_{1} \times$ $\mathrm{P}_{5}, \mathrm{P}_{3} \times \mathrm{P}_{12}$ and $\mathrm{P}_{9} \times \mathrm{P}_{11}$ for plant height; $\mathrm{P}_{2} \times \mathrm{P}_{6}, \mathrm{P}_{5} \times$ $\mathrm{P}_{12}, \mathrm{P}_{1} \times \mathrm{P}_{12}$ and $\mathrm{P}_{4} \times \mathrm{P}_{7}$ for number of pods per plant and pod yield per hectare; $\mathrm{P}_{2} \times \mathrm{P}_{6}, \mathrm{P}_{4} \times \mathrm{P}_{7}, \mathrm{P}_{5} \times \mathrm{P}_{12}, \mathrm{P}_{1}$ $\times \mathrm{P}_{3}$ and $\mathrm{P}_{1} \times \mathrm{P}_{12}$. The present results were in conformity to those of the earlier workers of okra like Singh et al. (2001), Sood and Kalia (2001) More and Patil (2003)and Lyngdoh et al.(2013) Patel and Patel (2016).

The extent of heterosis for five best crosses over better parent and standard check (48.32\% to $82.42 \%$ and $7.13 \%$ to $35.66 \%$, respectively) for yield per hectare suggested the great scope of realizing higher yield in okra through heterosis breeding. Other economic traits also recorded moderate to high level of heterosis over the better parents.

\section{Conclusion}

The hybrid $\mathrm{P}_{2}(\mathrm{IC}-282280) \times \mathrm{P}_{6}(\mathrm{EC}-329380)$ was found to be the most promising for pod yield and other desirable traits. This cross also showed high heterosis over better parent and standard check for pod yield $(82.42 \%$, and $35.66 \%)$ and number of pods per plant $(62.82 \%$ and $48.54 \%)$ respectively. It is also clear that the high degree of non additive gene action for all the component traits observed in the present study favours hybrid breeding methodology So, it can be identified as the potential okra hybrid combination for commercial exploitation.

\section{REFERENCES}

Arapitsas, P. (2008). Identification and qualification of Polyphenolic Compounds from okra seeds and skins. Food Chemistry, 110: 1041-1045

Dilruba, S., Hasanzzaman, M., Karim, R. and Nahar, K. (2009). Yield response of okra to different sowing time and application of Growth Hormones. Journal of Horticultural Science and Ornamental plants, 1: 10-14

Gopalan, C., Sastri, S. B. V. and Balasubramanian, S. (2007). Nutritive value of Indian foods, National Institute of Nutrition (NIN, ICMR) India.

Kahlon, T. S., Chapman, M. H. and Smith, G. E. (2007). In vitro binding of bile acid by okra beets asparagus eggplant turnips green beans carrots and cauliflower. Food Chemistry, 103: 676-680

Kempthorne, O. (1957). An introduction to genetical stastistics. Jhon weleyand sons (Eds.I). New yark Pp. 458-670

Lyngdoh, Y. A., Mulge, R. and Shadap, A.( 2013). Heterosis and combining ability studies in nearhomozygous lines of okra [Abelmoschus esculentus (L.) moench] for growth parameters. The Bioscan, 8(4):1275-1279

More, D. C. and Patil, H. S. (2003). Heterosis and inbreeding depression for yield and yield components in okra.Indian Journal of Agricultural Research, 31(3): 141-148

Owolarafe, O. K. and Shatonde, H. O. (2004). Some physical properties of okra fruit. Journal of food Engineering, 63:299-302

Panse, V. G. and Sukhatme, P. V. (1989). Statistical Methods for Agricultural Workesr, ICAR, New Delhi.

Patel, B. G. and Patel, A. I. (2016). Heterosis Studies in Okra (Abelmoschus esculentus (L.) Moench). Annals of 
Sujit Kumar et al. / J. Appl. \& Nat. Sci. 9 (2): 774 - 779 (2017)

Agricultural and Environmental Sciences, 1(1): 15-20

Saifullah, M. and Rabbani, M. G. (2009). Evaluation and characterizations of Okra [Abelmoschus esculentus (L.) Moench.] genotypes. SAARC Journal Agriculture, 7:92-99

Singh, R. K., Mandal, G. and Singh, V. K. (2001). Studies on combining ability in okra [Abelmoschus esculentus (L.)
Moench]. Progressive Horticulture, 21(1-2): 1-8

Sood, S. and Kalia, P. (2001). Heterosis and combining ability studies for some quantitative traits in okra [Abelmoschus esculentus (L.) Moench]. Haryana Journal of Horticultural Science, 30(1/2): 92-94

Student. (1908a). The probable error of a mean. Biometrika, 6:1-25 